\title{
CPTAC Prostate Adenocarcinoma Baseline
} Form

National Cancer Institute

\section{Source}

National Cancer Institute. CPTAC Prostate Adenocarcinoma Baseline Form. NCI

Thesaurus. Code C157530.

Terminology used in support of the data collection efforts of the Clinical Proteomic

Tumor Analysis Consortium (CPTAC) with the focus on Prostate Adenocarcinoma

Baseline Form data. 